\title{
SOBRE LA SUPERPOSICIÓN DE LOS CONCEPTOS DE 'CUANTIFICACIÓN' E ‘INTENSIFICACIÓN’ EN LA LINGÜÍSTICA
}

Resumen. Con este artículo se pretende hacer un primer acercamiento al fenómeno de la superposición de los conceptos de 'cuantificación' e 'intensificación', causa del frecuente uso indiferenciado de ambos. Un breve análisis de los significados y funciones otorgados por la lingüística a esas nociones conduce a revelar coincidencias denotativas de estas y el grado de su relación con factores pragmáticos.

Palabras clave: semántica, pragmática, cuantificación, intensificación.

\section{A modo de introducción}

El término de 'cuantificación' en la lingüística se ve a menudo confundido, cuando no igualado, con el de 'intensificación'. Seguramente, eso se debe a ciertas áreas semántico-funcionales de ambos fenómenos que resultan sobrepuestas hasta tal punto que a menudo es difícil separar el uno del otro, por no decir imposible.

En la literatura disponible no faltan ejemplos en los que distintos autores emplean de modo indiferenciado los dos términos, de manera que parecen sinónimos, lo cual, indudablemente, confirma la coexistencia de los fenómenos designados por estos conceptos.

Con el fin de hacer una primera aproximación a esa interesante coincidencia denotativa y destacar las principales causas de la frecuente confusión de ambos términos, nos hemos propuesto realizar un breve análisis de los respectivos significados y funciones que se les otorga en la lingüística actual, dedicando una atención especial a los logros de la lingüística hispánica en esta materia.

\footnotetext{
* Uniwersytet Szczeciński.
} 


\section{La cuantificación lingüística}

El término de 'cuantificación', cuyo significado léxico básico está relacionado con «expresar númericamente una magnitud de algo» (DLE, s.v. 'cuantificar'), tiene una presencia relativamente reciente en la lingüística. Según lo presentado por López Palma (1999: 81), los primeros estudios gramaticales dedicados a la cuantificación aparecen en la segunda mitad del siglo $\mathrm{XX}$, y esa entrada tardía en el ámbito de la lingüística seguramente se debe al hecho de que «en el lenguaje ordinario no es fácil identificar los elementos que son equivalentes a las unidades que forman la estructura (operador, viable) de la lógica, pues en el lenguaje natural se dan fenómenos de sincretismo, amalgamas, homonimia o sinonimia» (López Palma, 1999: 81). Valga añadir que desde el principio los estudios lingüísticos se enfocan sobre todo en la cuantificación de los nombres.

La aparición del término de 'cuantificación' en la lingüística, debida al creciente interés por estudiar el alcance referencial de los indefinidos, no supuso la creación de una nueva categoría gramatical. Puesto que la cuantificación es un fenómeno semántico, la noción de 'cuantificador', lógicamente, hace referencia a una propiedad semántica, compartida por diferentes categorías gramaticales, como, por ejemplo, los sustantivos, los adjetivos, los adverbios y los pronombres.

A pesar de su indudable carácter semántico, cabe mencionar que la cuantificación lingüística depende mucho de diferentes factores pragmáticos, siendo un hecho comunicativo que se manifiesta únicamente en la enunciación del discurso lingüístico (Lamíquiz, 1991: 29). Así, conviene subrayar que está relacionada con las circunstancias contextuales y cotextuales, los conocimientos pragmáticos de los interlocutores y, habitualmente, con la individual valoración apreciativa en subjetivas mediciones basadas en la experiencia personal (Lamíquiz, 1991: 29). Dicha valoración se comunica implícitamente, transmitiendo al mismo tiempo «la idea de magnitud, la impresión agradable o desagradable que se tenga de algo, la tendencia a favor o en contra de ello, la satisfacción o decepción que aquello pueda causar» (Kaul de Marlangeon, 2002: 133).

Dado que el lenguaje humano es un fenómeno de una extraordinaria complejidad, la cuantificación lingüística puede realizarse no solo respecto a individuos (cuantificación nominal), sino también a acciones (cuantificación verbal) y propiedades (cuantificación adjetival). Este hecho se viene subrayando desde hace algún tiempo, destacando que, a diferencia de las ciencias de la naturaleza, las «ciencias humanas [... ], y entre ellas la lingüística, centran todo su interés en lo cualitativo» (Lamíquiz, 1991: 18). 
Con eso se llega a evidenciar que la cuantificación lingüística sale más allá de una mera expresión de la cantidad de lo contable o mesurable, abarcando también el grado de toda aquella sustancia conceptual que es difícil de contar o medir ${ }^{1}$.

Esa visión muy amplia de lo que la lingüística pretende incluir bajo el nombre común de 'cuantificación' origina diversas distinciones tipológicas de este fenómeno, de las cuales especialmente las relacionadas con la cuantificación de sustancias conceptuales lexicalizadas en ciertas categorías gramaticales se aproximan mucho al fenómeno de intensificación. Precisamente las diferentes formas de presentarse la sustancia conceptual, o sea, los modos lógicos, son responsables de que los lingüistas opten por distinguir, grosso modo, dos tipos de cuantificación. Así, en primer lugar, se distingue la cuantificación numerada de las sustancias discontinuas, siguiendo el criterio de «multiplicidad o cuantificación externa en asociación sumativa» (Lamíquiz, 1991: 38); son los casos de los modos lógicos 'ejemplar' (He visto dos gatos negros) y de 'acción cíclica' (Leí este libro tres veces). Los modos lógicos mencionados permiten también una cuantificación no numerada, llamada interna, indefinida o de intensidad (Compraste demasiados plátanos; Su hijo la visita mucho).

Por su parte, la cuantificación interna es la única aplicable en el caso de los modos 'clase' (Compramos poca fruta), 'atributo' (Sois bastante listos) y 'acción no cíclica' (El ruido me molesta mucho), puesto que la naturaleza continua de estas formas de sustancia conceptual impide el empleo de la cuantificación numerada. La modalidad interna o intensiva de la cuantificación desde hace algún tiempo funciona principalmente bajo un nuevo término: 'cuantificación de grado'. Se observa que en este tipo de cuantificación los operadores ligan variables que no denotan individuos, sino niveles jerárquicamente ordenados en una escala, expresando la cantidad o intensidad de una determinada realidad o cualidad (Sánchez López, 1999: 1090).

En lo tocante a la escala en la que se posiciona la sustancia cuantificada, aquella «se sitúa entre el todo o cantidad máxima posible y la nada hacia donde se aproxima la sustancia en grado cuantitativo mínimo» (Lamíquiz, 1991: 49). Volvemos a destacar que son los factores pragmáticos los que permiten establecer la orientación de los operadores en la escala, de ahí que la interpretación final del valor de un conjunto cuantificado siempre dependa del contexto (Sánchez López, 1999: 1090).

${ }^{1}$ Identificar la cuantificación lingüística solo con los casos en los que se produce la extensión del nombre por la expresión de su número se considera una postura restringida, dada la existencia de otros elementos que son capaces de modificar cuantitativamente tanto SSNN como oraciones u otras categorías gramaticales (Sánchez López, 1999: 1028). 
Los medios lingüísticos capaces de expresar la cuantificación son diversos: desde los rasgos sémicos presentes en determinadas palabras (mar, enjambre) y la iteración lexemática (Entero, entero no lo he leído), hasta los afijos (extrafino, lujazo, arbolito) y morfemas gramémicos independientes (diez, poco, todo) (Lamíquiz, 1991: 56 y ss.), pero solo estos últimos funcionan en las gramáticas bajo el nombre de 'cuantificadores'2. Obviamente, la lista de los recursos lingüísticos de carácter cuantificador no constituye un grupo cerrado y eso, a nuestro modo de ver, es así por dos razones: la creatividad de los hablantes (manifiesta, sobre todo, en el léxico coloquial) y la imprecisión de los límites del propio fenómeno de cuantificación. Este último motivo está relacionado, como ya ha quedado plasmado en líneas anteriores, con el hecho de que la cuantificación lingüística es propensa a ir cargada de ponderación valorativa o intensiva, lo cual, en teoría, abre la posibilidad de incluir en la cuantificación prácticamente todas las maneras lingüísticas intensificadoras posibles.

\section{La intensificación}

El segundo de los términos objeto del presente artículo también ha tenido su entrada en la lingüística hace relativamente poco. De la información proporcionada por Straś (2008: 11-12) se deprende que los estudios sobre la intensificación fueron promovidos por aquellos dedicados a la gradación, iniciados de manera sistemática en los años setenta del siglo pasado (p. ej. Bolinger, 1972; Cresswell, 1977). La lingüista destaca que la intensificación también ha sido objeto de estudio de varios científicos polacos, los cuales iniciaron sus investigaciones en los años ochenta (Straś, 2008: 13), la década en que también surge el interés por este fenómeno en la lingüística española ${ }^{3}$.

La intensificación lingüística, que en diferentes trabajos del ámbito hispano aparecía bajo toda una serie de otras denominaciones ('énfasis', 'expresividad', 'ponderación', 'superlación'4, entre otros), suele

${ }^{2}$ En del grupo de cuantificadores las gramáticas incluyen ante todo los indefinidos, numerales cardinales y distribuitivos (RAE-ASALE, 2009: § 19.5m), llamados también 'cuantificadores propios', además de partículas focales (Sánchez López, 1999: 1105).

${ }^{3}$ Los estudios sobre la intensificación en la lengua española son particularmente desarrollados desde los años noventa por el grupo Val.Es.Co <www.valesco.es> de la Universidad de Valencia, en especial por Marta Albelda Marco y Antonio Briz Gómez.

4 Todos esos términos han surgido por el afán de explicar la finalidad de uso de la intensificación (Albelda Marco, 2014: 85). 
presentarse como un fenómeno de naturaleza más compleja que la de la cuantificación, tanto por transmitir numerosos matices expresivos como por el número de procedimientos lingüísticos empleados en su producción. Respecto a estos últimos, cabe subrayar que la intensificación se manifiesta a través de diferentes recursos a todos los niveles de la lengua: fónico, léxico-semántico, morfológico-sintáctico y discursivo. Además de los intensificadores lingüísticos, al repertorio de los medios utilizados por la intensificación cabe añadir todas las estrategias paraverbales y quinésicas.

En principio, a la intensificación también se le otorga naturaleza semántica ( $c f$. Straś, 2008: 15 y ss.), destacando que este fenómeno tiene alcance más amplio que la cuantificación, la cual se suele considerar como una de las numerosas maneras de intensificar. Entonces, todo caso de intensificación, en primer lugar, recibe una descripción semántica en la que se tiene que dar cuenta de cómo este valor se codifica en la lengua, anunciando y revelando una propiedad muy importante en la identificación del fenómeno en cuestión: la escalaridad. Las escalas que pueden someterse al proceso de intensificación en el nivel semántico son varias, a saber: la de la cuantificación (expresada por los numerales cardinales y los indefinidos), la de la dimensión (que suele manifestarse por el uso de adjetivos pertinentes) y la del grado de intensidad de una propiedad (codificada en ciertos adjetivos, adverbios, verbos y diversos tipos de locuciones) (Mihatsh y Albelda Marco, 2016: 8-9). Las escalas de modalidad deóntica y epistémica (expresadas por varios adverbios modales y marcadores discursivos) se sitúan a medio camino entre la semántica y la pragmática (Mihatsh y Albelda Marco, 2016: 9).

Evidentemente, quedarse tan solo con la perspectiva semántica de la intensificación no es la postura adecuada, ya que eso supondría aceptar que «la intensificación se encuentra a priori codificada en determinados mecanismos lingüísticos» (Albelda Marco, 2014: 82). Además, la intensificación no solo es un valor de significado, sino, sobre todo, una estrategia de comunicación que se emplea para un fin concreto, ergo, en unas determinadas circunstancias contextuales (Albelda Marco, 2014: 82). Así, ha de destacarse, en primer lugar, el carácter estratégico de la intensificación, que busca la efectividad y la eficiencia en el habla, y, por otra parte, la importancia del contexto, sin cuyo análisis no sería posible identificar la intención intensificadora en determinados recursos lingüísticos (Albelda Marco, 2014: 82).

Reconocer la naturaleza eminentemente pragmática de la intensificación ${ }^{5}$ ha llevado a los investigadores a distinguir las siguientes

${ }^{5}$ En el marco de la lengua española, ese reconocimiento se produjo gracias al trabajo de Meyer-Hermann (1988). 
escalas pertenecientes a este nivel: la de la fuerza ilocutiva, la del grado de cortesía y la de grados de afectividad y expresividad (Mihatsh y Albelda Marco, 2016: 9). Esos nombres de diferentes escalas pragmáticas son sustuidos por los investigadores de Val.Es.Co con los términos de atenuación e intensificación del decir ${ }^{6}$, que sirven para referirse a todos los fenómenos de intensidad a nivel pragmático (Mihatsh y Albelda Marco, 2016: 9).

Aparte del concepto de 'escalaridad', de vital importancia para establecer el grado de intensificación, hay que destacar el de 'evaluación', que «pretende servir como criterio de reconocimiento de la intensificación» (Albelda Marco, 2014: 85). Siendo la evaluación un rasgo relacionado con la actitud del hablante ante lo dicho, se encuentra en el juicio asociado a la información transmitida. Seguramente, en toda la teoría referente a la intensificación, ese es el eslabón más importante, ya que viene determinado por todo lo relativo a la connotación semántica y hace posible descubrir el carácter pragmático de la intensificación.

El peso del aspecto valorativo en la intensificación es innegable, puesto que «Quien intensifica, no solo expone un estado de cosas, sino que juzga la realidad, y con la elección de las formas lingüísticas pretende revelar este juicio.» (Albelda Marco, 2014: 85). Ahora bien, el juicio que se produce en la intensificación gracias a determinados valores manifiestos a través de las formas lingüísticas utilizadas es, como cabe de esperar, subjetivo. Entonces, es la subjetividad, que va unida a la evaluación, la que parece desempeñar el papel decisivo en la distinción entre los dos conceptos aquí tratados, porque «mientras que la cuantificación supone expresar que algo aumenta o disminuye su valor, la intensificación supone que el hablante transmite su punto de vista» (Albelda Marco, 2014: 85).

Con todo eso, distinguir los enunciados subjetivos de aquellos que no lo son sería una tarea ardua y un tanto artificial, puesto que cada enunciado se produce dentro de ciertas coordenadas espacio-temporales y psíquico-sociales, lo cual hace prácticamente imposible deshacerse del punto de vista subjetivo del hablante. Por eso, opinamos que no se dan muestras lingüísticas que puedan ser calificadas únicamente como cuantitativas, sino que siempre deberían verse relacionadas con alguna información de carácter pragmático, permitiendo de esta manera detectar la presencia de la intensificación.

${ }^{6}$ Ambos términos se utilizan para referirse al procedimiento interactivo en el cual los hablantes, empleando operadores de intensificación/atenuación, asumen y/o establecen con respecto a una proposición $p$ un grado de intensidad obligativa (Meyer-Hermann, 1988: 283). 


\section{Observaciones finales}

Según se ha podido observar en líneas anteriores, los dos conceptos aquí comentados poseen algunos puntos de contacto, que hacen que se produzca cierto tipo de semejanza entre ellos. Así, en principio, ambos se pueden estudiar desde la perspectiva semántica, denotando fenomenos cuyos valores se caracterizan por ser escalares.

No obstante, aunque a ambas nociones se les otorga naturaleza semántica, esta es esencial y constitutiva de la cuantificación, y solo un punto de partida para los estudios enfocados en la intensificación, los cuales tienden hacia la pragmática.

Precisamente ese carácter preponderantemente pragmático es lo que diferencia más la intensificación de la cuantificación, ya que en los estudios dedicados a esta última los aspectos pragmáticos se aprovechan solo para la adecuada interpretación de subjetivas mediciones basadas en la experiencia personal. En cambio, el enfoque pragmático es imprescindible para caracterizar la intensificación, puesto que esta se refiere a una estrategia comunicativa, orientada a un fin, y que se infiere del modo de expresión elegido por el hablante, quien expresa así su evaluación subjetiva.

Retomando el tema relacionado con la semántica, hay que destacar que la confusión de ambos términos se debe también a la borrosidad del ámbito referencial del término de 'cuantificación', cuyo alcance denotativo, en principio, suele considerarse menor que el de la noción de 'intensificación'. No obstante, aunque los lingüistas, por un lado, tratan de limitar el papel de la cuantificación al mero hecho de medir y expresar una magnitud, por otro lado, al ampliar el alcance de este término, haciéndolo aplicable también a las sustancias conceptuales de carácter continuo, se han visto obligados a admitir el carácter intensivo del fenómeno designado por él. Y este es, posiblemente, el origen de mayor confusión de los dos términos, ya que la cuantificación intensiva o de grado supone denotar sobre todo la intensidad de una realidad o cualidad, o sea, su valor denotativo en ese momento es prácticamente igual al de la intensificación estudiada desde la perspectiva semántica.

Finalmente, creemos necesario subrayar que aunque la cuantificación se presenta en numerosas fuentes como un fenómeno relacionado ante todo con la semántica, es muy difícil que se vea desprovista de ninguna información de carácter pragmático y, por ende, que no coaparezca en un enunciado junto con la intensificación. Es un hecho más que explica la frecuente confusión de los dos términos estudiados en el presente artículo. 


\section{Bibliografía}

ALBELDA MARCO, M. (2014). «Escalaridad y evaluación: rasgos caracterizadores de la intensificación pragmática», en E. PUSTKA y S. GOLDSCHMITT (eds.), Emotionen, Expressivität, Emphase. Berlin: Erich Schmidt Verlag, 79-94.

BOLINGER, D. (1972). Degree Words. The Hague-Paris: Mouton.

CRESSWELL, M. J. (1977). «The semantics of degree», en B. PARTEE (ed.) Montague grammar. New York: Academic Press, 261-292.

KAUL DE MARLANGEON, S. B. (2002). Los adverbios en -mente del español de hoy y su función semántica de cuantificación. Madrid: Iberoamericana.

LAMÍQUIZ, V. (1991). La cuantificación lingüística y los cuantificadores. Madrid: UNED.

LÓPEZ PALMA, H. (1999). La interpretación de los cuantificadores. Aspectos sintácticos y semánticos. Madrid: Visor Libros.

MEYER-HERMANN, R. (1988). «Atenuación e intensificación (análisis pragmático de sus formas y funciones en español hablado)», Anuario de Estudios Filológicos, XI, 275-290.

MIHATSH, W. y ALBELDA MARCO, M. (2016). «Introducción. La atenuación y la intensificación desde una perspectiva semántico-pragmática», RILI-Revista internacional de lingüística iberoamericana, XIV, 7-18.

RAE-ASALE, (2009). Nueva gramática de la lengua española. Madrid: Espasa.

242 SÁNCHEZ LÓPEZ, C. (1999). «Los cuantificadores: clases de cuantificadores y estructuras cuantificativas», en V. DEMONTE y I. BOSQUE (coord.), Gramática descriptiva de la lengua española, vol. 1, 1025-1128.

STRAŚ, E. (2008). Kategoria intensywności we frazeologii języka polskiego i rosyjskiego. Katowice: Wydawnictwo Uniwersytetu Śląskiego.

\section{Diccionario}

DLE - Diccionario de la Lengua Española [en linea] <www.dle.rae.es>, fecha de consulta: 3 de abril de 2018. 